\title{
Reactividad del Aminal Macrociclico1:3,7:9,13:15,19:21- TetrametILENTETRAHEXAHIDROPIRIMIDINA (TMTP) con Fenoles P-Sustituidos
}

\author{
ReActivity OF MACROCYCLIC 1:3,7:9,13:15,19:21- \\ TETRAMETILENTETRAHEXAHIDROPIRIMIDINA AMINAL (TMTP) \\ WITH P-SUBSTITUTED PHENOLS
}

\section{Alcázar Franco Daniel Jesús', Herrera Herrera Claudia Patricia², Fuentes Gandara Fabio Armando ${ }^{3}$}

\author{
1 Universidad de la Costa, Barranquilla, Colombia, dalcazar1@cuc.edu.co \\ 2 Universidad de la Costa, Barranquilla, Colombia, cherrera8@cuc.edu.co \\ ${ }^{3}$ Universidad de la Costa, Barranquilla, Colombia, f fuentes1@ cuc.edu.co
}

\section{RESUMEN}

Una nueva serie de compuestos de estructuras tipo 1,3-hexahidropirimidina se logró sintetizar, con el fin de comprobar y ratificar la reactividad del aminalmacrociclico: 1:3,7;9,13;15,19;21-tetrametilentetrahexahidropirimidina (TMTP) con fenoles para-sustituidos, empleando reacciones tipo Mannich en medio alcalino. Las estructuras se elucidaron por métodos espectroscópicos, las cuales mostraron compuestos consistentes con las estructuras propuestas. Los compuestos sintetizados no se encuentran reportados en la literatura consultada.

Palabras clave: bases di-Mannich, hexahidropirimidina, macrociclo, sustituido fenol

\section{ABSTRACT}

A new series of compounds 1,3-type structures was achieved hexahydropyrimidine synthesize, checking and confirming the reactivity of 1:3,7,9.13,15.19,21-tetrametilentetrahexahidropirimidina (TMTP) with for-substituted phenols, using Mannich type reaction in alkaline medium, structures were elucidated by spectroscopic methods, compounds which showed consistent with the proposed structures. The synthesized compounds are not reported in the literature.

keywords: di-Mannich base, hexahydropyrimidine, macrocycle, substituted phenol 


\section{INTRODUCCIÓN}

El término aminal fue introducido en 1956 para designar los equivalentes aminados de acetales y mercaptales. Son gem-diaminas que se caracterizan por la presencia de dos grupos di o mono sustituidos en el mismo átomo de carbono, los cuales le confieren propiedades químicas especiales en reacciones, frente a núcleofilos.

Pueden ser de cadena abierta como la metilendiamina [1] o ncíclicos como la 1,3-imidazolidina [2] o la 1,3-hexahidropirimidina [3]. Cabe anotar que la denominación cíclica se emplea cuando la función aminal hace parte de un heterociclo.

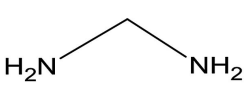

1

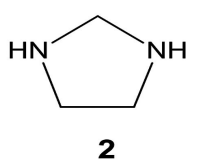

2

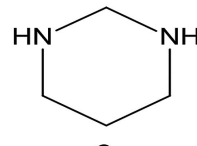

3
Cuando dicha función está presente en ciclos más grandes o más complejos, que involucran varios sistemas anulares, tales como la hexametilentetramina (Urotropina) [4], el 1,3,6,8 tetrazatriciclo[4,4,1,13,8]dodecano (TATD) [5], y la 1:3,7:9,13:15,19:21-tetrametilentetrahexa-hidropirimidina (TMTP) (6) [1], [2], [7], se les conoce como aminales macrocíclicos.

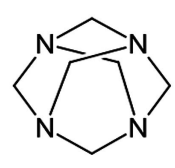

4

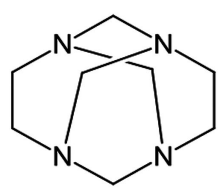

5
6

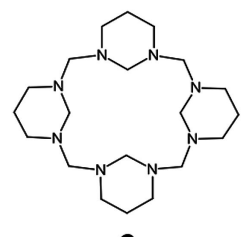

La química y modo de reacción de estos amínales, aunque es muy interesante, no se han estudiado mucho. Se conocen estudios que describen una condensación tipo Mannich en medio alcalino entre [5] y fenoles [3], [8]. Dichos estudios han demostrado que estos compuestos pueden considerarse como agentes amino metilantes. Si observamos en el esquema 1, la reacción general de Mannich involucra la combinación de un aldehído, generalmente formaldehido, una amina y un compuesto que presenta hidrógenos activos, produciendo una amino metilación sobre dichos compuestos y generando, de esta manera, la base de Mannich [4].

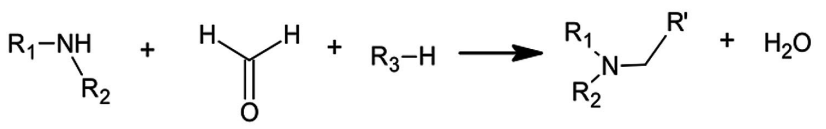

Fig. 1 Reacción general de Mannich

El tamaño del sistema anular y la forma general de la molécula son factores importantes en la reactividad química de los amínales cíclicos. Para el caso del 1:3,7:9,13:15,19:21-tetrametilentetrahexahidropirimidina (TMTP) (6), obtenido a partir de la condensación de formaldehido con 1,3-propanodiamina [5], los pocos trabajos que se conocen en cuanto a su reactividad química, han sido desarrollados por el grupo de investigación en Síntesis Orgánica de la Universidad Nacional de Colombia, destacándose el estudio que involucra la reacción de 6 con fenoles "para"-sustituidos como: el $p$-cresol, $p$-clorofenol y el fenol [1], [2].

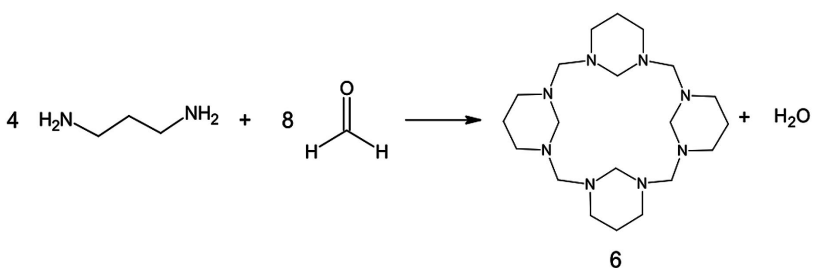

Fig. 2 Síntesis de "TMTP"

Los productos de estas reacciones son compuestos heterocíclicos del tipo: 2,2'-(dihidropirimidina-1,3(2H,4H)-dimetanodiil)bis(4-sustituidosfenoles) o también los conocidos como 1,3-bis-(hidroxibencil) hexahidropirimidinas [8], cuya estructura corresponde a bases tipo diMannich. Los compuestos sintetizados no se encuentran reportados en la literatura. 


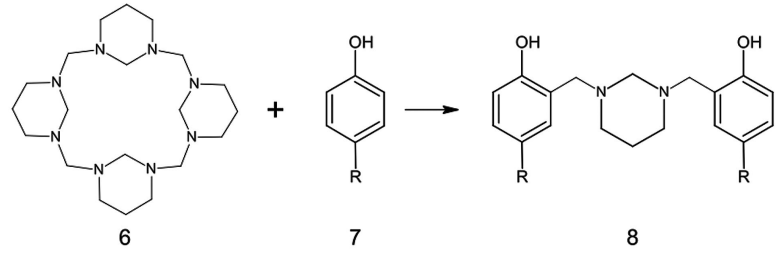

Fig. 3 Reacción general de "TMTP" con fenoles p-sustituidos

\section{Resultados y Discusión}

Empleando reacciones tipo Mannich en medio alcalino entre el aminal macrocíclico 1:3,7;9,13;15,19;21-tetrametilentetrahexahidropirimidina (TMTP) con fenoles para-sustituidos, se logró comprobar la reactividad del (TMTP) y sintetizar, al igual que en trabajos anteriores, bases di Mannich del tipo 1,3-hexahidropirimidina [8], utilizando fenoles específicos (7a; 7b y 7c).

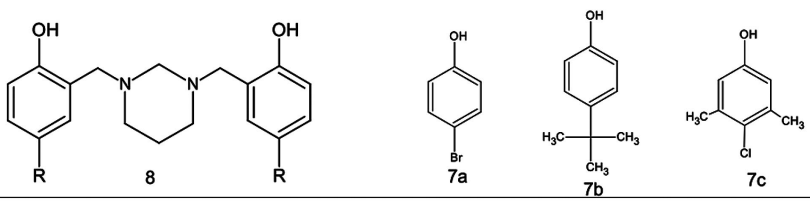

La reactividad química del (TMTP) con fenoles p-sustituidos fue estudiada previamente por [5], como también por el grupo de investigaciones de compuestos heterocíclicos de la Universidad Nacional de Colombia y en todos los casos se obtuvieron bases di-Mannich del tipo 1,3-hexahidropirimidina [8]; en este trabajo, además de la reactividad, también se evaluó el efecto del volumen estérico del sustituyente, utilizando fenoles que presentaban un mayor impedimento a los ya trabajados, como son el $p$-bromofenol (7a), el $p$ terbutilfenol (7b), y el 4-cloro-3,5-dimetilfenol (7c). Los resultados ponen en evidencia, que muy a pesar de la existencia de variaciones en el pH del medio de reacción, provocadas por los diferentes grados de acidez de los fenoles, estas no son lo suficiente como para afectar el transcurso de la misma. Inicialmente, los ensayos preliminares, siguiendo la metodología descrita previamente [2], [3], no produjeron los rendimientos ni los resultados esperados, por lo que fue necesario realizar modificaciones a las condiciones de reacción.
Primero, se estudió el efecto del orden de adición de los reactantes y, posteriormente, la temperatura de reacción. El orden de adición no tuvo ningún efecto neto sobre la reacción, pero se pudo comprobar que la temperatura óptima de reacción se da bajo condiciones de reflujo, y el tiempo de reacción es variable y característico para cada uno de los fenoles trabajados. Los diferentes tiempos y rendimientos de reacción tienen relación con lo informado por Santafé [6], quien atribuye estas diferencias al grado de activación del anillo aromático frente a la sustitución electrofilia, efecto que gobierna la mayor formación de productos. Sin embargo, en cuanto a los análisis espectroscópicos se logra concluir que están acordes con los observados en los fenoles ya trabajados por el grupo de investigación de heterocíclicos de la Universidad Nacional [10], [15], aunque es importante profundizar aún más en la investigación.

Los productos obtenidos difieren únicamente en la naturaleza de los sustituyentes presentes en el anillo aromático. Por tanto, la discusión sobre su identidad se realizó de forma general, tomando como referencia los análisis efectuados anteriormente, y abordando solamente aspectos que diferenciaban y complementaban su asignación estructural.

En los espectros $\mathrm{RMN}^{1} \mathrm{H}$ y $\mathrm{RMN}^{13} \mathrm{C}$ para las bases di-Mannich [1] obtenidas, solo se observan pequeñas diferencias en los desplazamientos químicos, efecto ocasionado por la naturaleza del sustituyente en la posición 4 del anillo aromático; en la región de protones aromáticos, los espectros RMN ${ }^{1} \mathrm{H}$ muestran señales características de un sistema de acoplamiento ABX, con desplazamiento y multiplicidades similares a las ya publicadas en la literatura. En la región de protones alifáticos, en el espectro $\mathrm{RMN}^{1} \mathrm{H}$, aparecen 4 señales que van desde 1,78; 2,73; 3,49; 3,70 ppm características de las bases di-Mannich, las cuales fueron corroboradas con los análisis de correlación en los experimentos Espectroscopia 2D de Correlación Heteronuclear (HMQC y HMBC), en 
los que se pudo observar menor dispersión en los desplazamientos químicos. Con estos resultados se logró concluir que en la obtención de las bases di-Mannich de los fenoles específicos (7a y 7c) no existe ninguna influencia desde el punto de vista del volumen estérico del sustituyente en el curso de la reacción y que esta debe seguir un mecanismo parecido al propuesto inicialmente Fig. 4.<smiles>[R]c1ccc(O)c(CN2CCCN(Cc3cc([R])ccc3O)C2)c1</smiles><smiles></smiles>

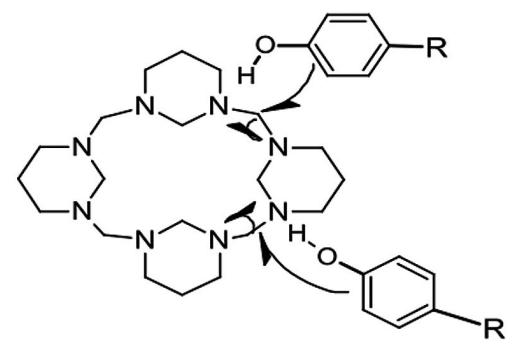

Fig. 4 Mecanismos de reacción de las bases di-Mannich de los fenoles específicos [13], 15], [16],

TABLA I

Condiciones de Reacción y Resultados Obtenidos

\begin{tabular}{|c|c|c|c|c|c|}
\hline $\begin{array}{c}\text { Fenol } \\
\text { (g) }\end{array}$ & $\begin{array}{c}\text { TMTP } \\
\text { (g) }\end{array}$ & $\mathbf{p H}$ & $\begin{array}{c}\text { Tiempo } \\
\text { (h) }\end{array}$ & $\begin{array}{c}\text { Producto } \\
\text { p.f }{ }^{\circ} \mathbf{C}\end{array}$ & $\begin{array}{c}\text { Rend } \\
(\%)\end{array}$ \\
\hline $\begin{array}{c}-\mathrm{Br} \\
0,86\end{array}$ & 0,47 & 8,5 & 14 & $8^{a}(158-160)$ & $21_{2}, 08 \%$ \\
\hline $\begin{array}{c}\text {-t-But } \\
0,75\end{array}$ & 0,47 & 8,0 & 12 & $8 b(188-190)$ & $25,66 \%$ \\
\hline $\begin{array}{l}\text { 4-cloro-3,5- } \\
\text { dimetilfenol } \\
0,78\end{array}$ & 0,47 & 8,0 & 15 & $8 c(204-206)$ & $27,09 \%$ \\
\hline
\end{tabular}




\section{Análisis Experimental}

\section{A. General}

A una solución del fenol correspondiente (7a-c) $\left(5,0 \times 10^{-3}\right.$ moles) en etanol al $96 \%$ ( $5 \mathrm{~mL}$.) bajo condiciones de reflujo, se le adicionó lentamente TMTP $\left(1,2 \times 10^{-3}\right.$ moles) disuelto en etanol al $96 \%$ (5 mL.). Una vez terminada la adición, la mezcla de reacción se dejó en reflujo por un tiempo variable y característico para cada fenol. Concluida la reacción, se suspendió el reflujo y se colocó en baño de hielo hasta la aparición de un precipitado, el cual se filtró y se secó. Una vez seco, se purificó por columna.

Así se obtuvo:

1. 2,2'-(dihidropirimidina-1,3(2H,4H)dimetanodiil)bis(4-bromofenol) (8a)
Sólido amarillo en forma de agujas con punto de fusión de $158-160^{\circ} \mathrm{C}$, soluble en diclorometano, cloroformo, etanol, metanol y benceno, e insoluble en hexano y agua. Se obtuvo con un rendimiento de $21,08 \%$. $R M N-{ }^{-1} \mathrm{H}\left(\mathrm{CDCl}_{3}, 400 \mathrm{MHz}\right)$ $\delta=3,73\left(\mathrm{~s}, 4 \mathrm{H}, \mathrm{Ar}-\mathrm{CH}_{2}-\mathrm{N}\right) ; 3,37\left(\mathrm{~s}, 2 \mathrm{H}, \mathrm{N}-\mathrm{CH}_{2}-\mathrm{N}\right)$; 2,73 (t, $4 \mathrm{H} ; \mathrm{CH}_{2}-4$ y $\left.\mathrm{CH}_{2}-6\right) ; 1,80\left(\mathrm{~m}, 2 \mathrm{H}, \mathrm{CH}_{2}-5\right)$; $7,22\left(\mathrm{dd}, 2 \mathrm{H}\right.$, sistema $A B X, \mathrm{~J}_{A B}=1,20 \mathrm{~Hz}, \mathrm{~J}_{A X}=$ $\left.8,58 \mathrm{~Hz}, \mathrm{H}-4^{\prime}\right) ; 7,01\left(\mathrm{~d}, 2 \mathrm{H}\right.$, sistema $\mathrm{ABX}, \mathrm{J}_{\mathrm{AB}}=$ $\left.2,62 \mathrm{~Hz}, \mathrm{H}^{\prime} 6^{\prime}\right) ; 6,72\left(\mathrm{~d}, 2 \mathrm{H}\right.$, sistema $A B X ; J_{B X}=$ $\left.8,57 \mathrm{~Hz}, \mathrm{H}-3^{\prime}\right) \cdot \mathrm{RMN}^{-13} \mathrm{C}\left(\mathrm{CDCl}_{3}, 100 \mathrm{MHz}\right) \delta=$ 156,9 (C2'), 131,0 (C6'); 130,5 (C4'); 121,5 (C5'); $117,3\left(\mathrm{C}^{\prime}\right) ; 110,2\left(\mathrm{C}^{\prime}\right) ; 71,8\left(\mathrm{NCH}_{2} \mathrm{~N}\right) ; 56,2$ $\left(\mathrm{CH}_{2} \mathrm{Ar}\right) ; 50,4$ (C4 y $\left.\mathrm{C} 6\right) ; 20,8$ (C5).

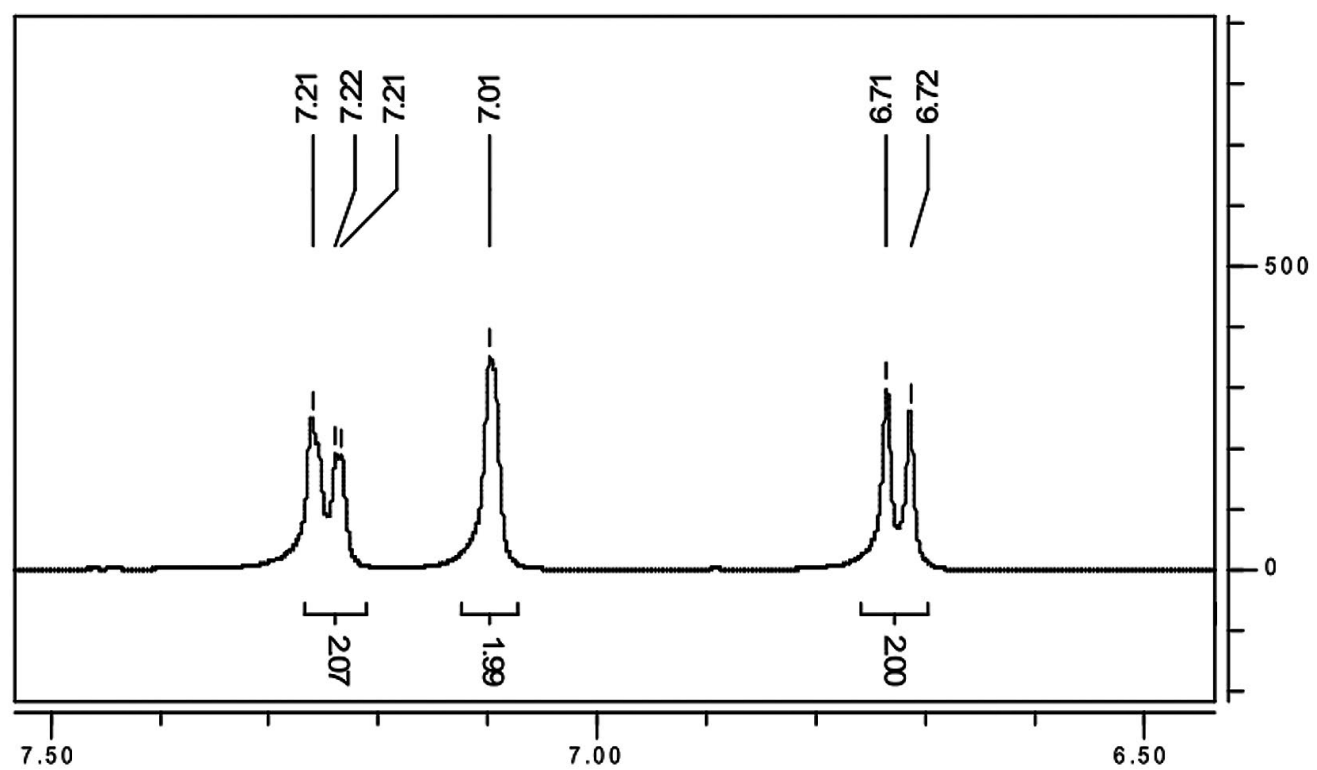

Fig. 5 Espectro RMN1H de 2,2'- (dihidropirimidina-1,3-dimetanodiil) bis (4- terbutilfenol) [9], [14].

2. 2,2'-(dihidropirimidina-1,3(2H, 4H)-dimetanodiil)bis(4-tert-butilfenol) (8b)

Sólido blanco con punto de fusión de 188$190^{\circ} \mathrm{C}$, soluble en diclorometano, cloroformo, etanol, metanol y hexano, e insoluble en benceno y agua. Se obtuvo con un rendimiento de $25,66 \%$. RMN ${ }^{-1} \mathrm{H}\left(\mathrm{CDCl}_{3}, 400 \mathrm{MHz}\right) \delta=3,79$ (s, $\left.4 \mathrm{H}, \mathrm{Ar}-\mathrm{CH}_{2}-\mathrm{N}\right) ; 3,49$ (s, $\left.2 \mathrm{H}, \mathrm{N}-\mathrm{CH}_{2}-\mathrm{N}\right) ; 2,73$ (t,
$4 \mathrm{H}, \mathrm{CH}_{2}-4$ y $\left.\mathrm{CH}_{2}-6\right) ; 1,78\left(\mathrm{~m}, 2 \mathrm{H}, \mathrm{CH}_{2}-5\right) ; 1,27$ (s, 18H, t-But); 7,18 (dd, 2H, ABX; Jab = 1,22 $\mathrm{Hz}$, Jax = 8,56 Hz, H-4'), 6,97 (d, 2H, sistema $\left.A B X, J x a=8,56 \mathrm{~Hz}, \mathrm{H}^{\prime} 3^{\prime}\right), 6,77$ (d, 2H, ABX; Jab $\left.=2,63 \mathrm{~Hz}, \mathrm{H}-6^{\prime}\right) ; \mathrm{RMN}^{-13} \mathrm{C}\left(\mathrm{CDCl}_{3}, 100 \mathrm{MHz}\right)$ $\delta=154,9$ (C2'), 141,7 (C6'); 125,3 (C4'); 125,2 (C5'), 119,5 (C1'); 115,3 (C3'); 73,1 ( $\left.\mathrm{NCH}_{2} \mathrm{~N}\right)$; $57,7\left(\mathrm{CH}_{2} \mathrm{Ar}\right) ; 50,7$ (C4); 33,8 (C(Me) $\left.)_{3}\right) ; 31,2$ $\left(\mathrm{C}\left(\mathrm{CH}_{3}\right)_{3}\right) ; 21,0(\mathrm{C} 5)$. 


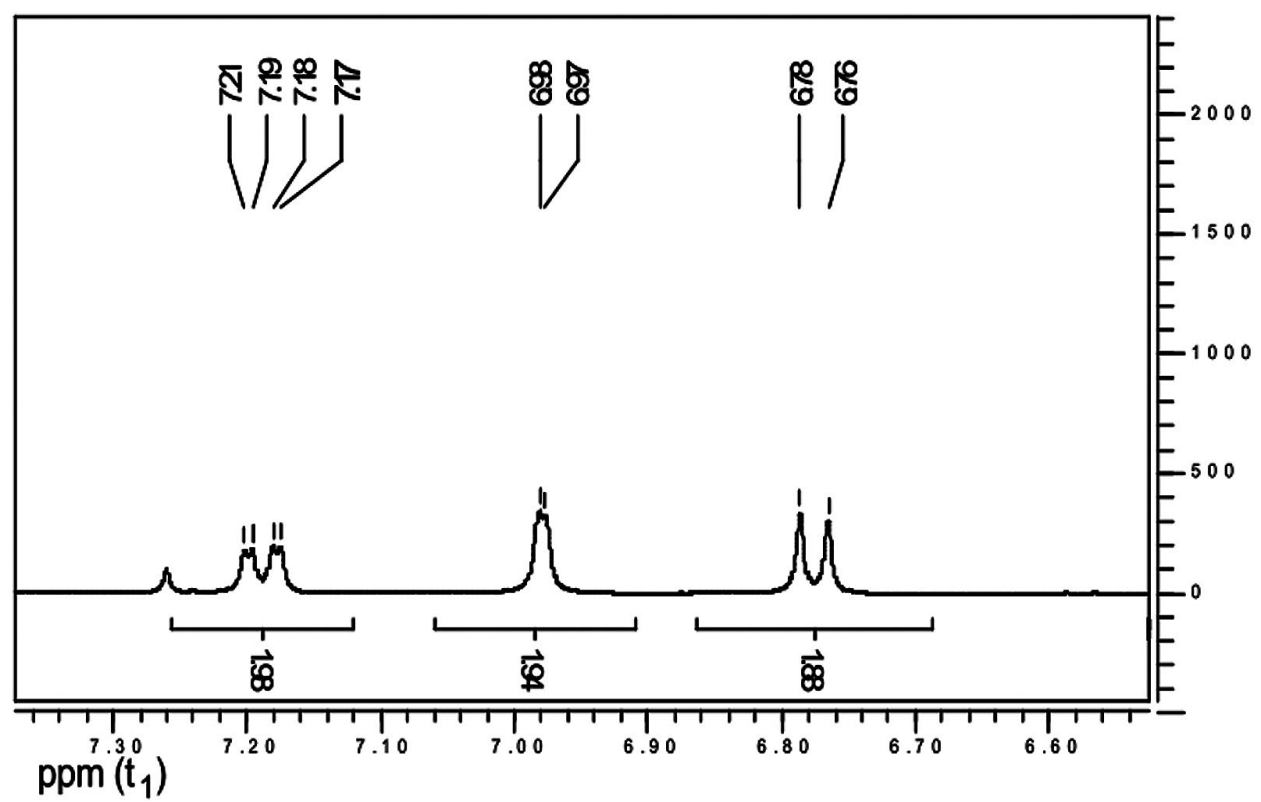

Fig. 6 Espectro RMN1H de 2,2'- (dihidropirimidina-1,3-dimetanodiil) bis (4- terbutilfenol) [9], [14].

3. 2,2'-(dihidropirimidina-1,3(2H,4H)-dimetanodiil)bis(4-cloro-3,5-dimetilfenol) (8c)

Sólido blanco de forma irregular con punto de fusión entre $204-206^{\circ} \mathrm{C}$, soluble en diclorometano, etanol, metanol, cloroformo y benceno, insoluble en hexano y agua. Se obtuvo con un rendimiento del $27,09 \%$. $\mathrm{RMN}^{-1} \mathrm{H}\left(\mathrm{CDCl}_{3}\right.$,
$400 \mathrm{MHz}) \delta=3,81$ (s, 4H, Ar- $\left.\mathrm{CH}_{2}-\mathrm{N}\right) ; 3,43$ (s, $\left.2 \mathrm{H}, \mathrm{N}-\mathrm{CH}_{2}-\mathrm{N}\right) ; 2,69\left(\mathrm{t}, 4 \mathrm{H}, \mathrm{CH}_{2}-4\right.$ y $\left.\mathrm{CH}_{2}-6\right) ; 2.31$ (s, $\left.6 \mathrm{H}, \mathrm{CH}_{3}-\mathrm{C}^{\prime} \circ \mathrm{CH}_{3}-\mathrm{C6}^{\prime}\right) ; 2,30\left(\mathrm{~s}, 6 \mathrm{H}, \mathrm{CH}_{3}-\right.$ $\mathrm{C}^{\prime}$ ○ $\left.\mathrm{CH}_{3}-\mathrm{C6}^{\prime}\right) ; 1,78\left(\mathrm{~m}, 2 \mathrm{H}, \mathrm{CH}_{2}-5\right) ; 6,62(\mathrm{~s}$, 2H). $R M N-{ }^{13} \mathrm{C}\left(\mathrm{CDCl}_{3}, 100 \mathrm{MHz}\right) \delta=156,2\left(\mathrm{C}^{\prime}\right)$, 136,6(C6'); 134,0 (C4'); 125,3 (C5'); 116,5 (C1'); 73,2 (C5); 54,0 (C4 у C6); 50,9 ( $\left.\mathrm{CH}_{2} \mathrm{Ar}\right) ; 21,0$ (C2); 16,6 $\left(\mathrm{CH}_{3}\right)$.

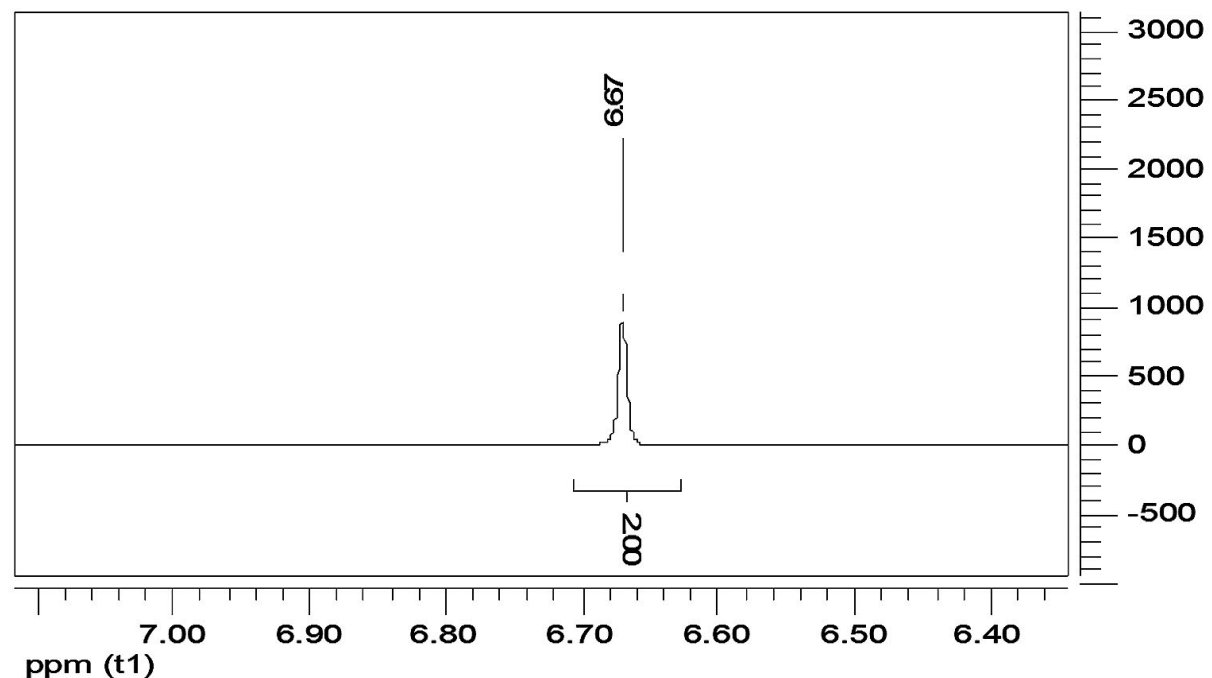

Fig. 7 Espectro RMN1H de 2,2'- (dihidropirimidina-1,3-diildimetanodiil) bis(4-cloro-3,5-dimetifenol) [9], [14]. 


\section{Conclusiones}

Al terminar esta investigación se llegó a las siguientes conclusiones:

- Se logró comprobar y confirmar la reactividad de aminal macrocíclico 1:3,7:9,13:15,19:21-tetrametilentetrahexahidropirimidina "TMTP"con fenoles $p$-sustituidos.

- El aminal macrocíclico1:3,7:9,13:15,19:21-tetrametilentetrahexahidropirimidina "TMTP" al reaccionar con fenoles $p$-sustituidos actúa como un agente aminometilante.

- La reacción del aminal macrocíclico 1:3,7:9,13:15,19:21-tetrametilentetrahexahidro pirimidina“TMTP" con fenoles $p$-sustituidos de mayor volumen estérico, da origen a compuestos del tipo 2,2'-(dihidropirimidina-1,3(2H,4H)dimetanodiil)bis(4-sustituidosfenoles).

- Se logró obtener la base di Manich: 2,2'-(dihidropirimidina-1,3(2H,4H)-dimetanodiil)bis (4-bromofenol).

- Se logró obtener la base di Manich:2,2'(dihidropirimidina-1,3 $(2 \mathrm{H}, 4 \mathrm{H})$-dimetanodiil)bis (4-terbutilfenol).

- Se logró obtener la base di Manich:2,2'(dihidropirimidina-1,3 $(2 \mathrm{H}, 4 \mathrm{H})$-dimetanodiil)bis (4-cloro-3,5-dimetilfenol).

- Se continúa analizando la reacción del amina Imacrocíclico1:3,7:9,13:15,19:21-tetrametilentetrahexahidropirimidina"TMTP" con el $p$-terbutilfenol, ya que se logró separar una segunda molécula en la reacción a la cual se le están realizando elucidaciones de sus propiedades.

\section{Sugerencias}

Realizar estudios para definir las posibles rutas mecanísticas alternas para la reacción entre el aminal macrocíclico tetrametilentetra hexahidropirimidina "TMTP" y el $p$-terbutilfenol, reacción en la cual se logró obtener otro producto de interés químico, aún no identificado.
Determinar la acción farmacológica de las sustancias sintetizadas en este trabajo.

Continuar el análisis entre el aminal macrocíclico tetrametilentetra hexahidropirimidina "TMTP" y el $p$-terbutilfenol, para determinar la importancia del otro producto que se obtuvieron en la reacción.

\section{Agradecimientos}

Agradecimientos por la realización y culminación de este proyecto en primera instancia a Dios, por no dejar desfallecer al grupo de investigación de Química de la Universidad Nacional encabezado por el doctor Jaime Ríos Motta, Director de este proyecto; a él gracias por toda su paciencia y su apoyo incondicional; al doctor Augusto Rivera, por toda su perseverancia y aportes y a la Universidad de la Costa, en cabeza del doctor Dixon Salcedo, por abrirnos las puertas para aportar al conocimiento.

\section{RefERENCIAS}

[1] W. Chaparro, W. y W. Alfonso, Reacciones de los Amínales MacrocíclicosHexametilentetramina y Tetrametilentetrahexahidropirimidina con Fenoles. Trabajo de grado, Farmacia, Universidad Nacional de Colombia, 1993.

[2] M. Rospenk, L. Sobczyk, A. Rabold, G. Zundel, Spectrochim. Act Part A, pp 55, 855, 1999.

[3] A. Rivera, G. I Gallo,. M. E Gayon,. P. Joseph-Nathan; Synth Commun, pp 23, 2921, 1993.

[4] P. Murray, B. Acta Cryst., pp 31, 583, 1975.

[5] M. Bejarano, Reacciones tipo Mannich del AminalMacrocíclicotetrametilentetra- hexahidropirimidina en Medio Alcalino. Tesis de Grado, Farmacia, Universidad Nacional de Colombia, 1993.

[6] G. Gallo, Síntesis y Reactividad Química de Algunos Amínales Macrocíclicos y su Participación en la Reacción de Mannich en Medio Básico. Tesis Doctoral en Ciencias Química, Universidad Nacional de Colombia, 1996.

[7] O. Muñoz,. Reacción de 1:3,7:9,13:15,19:21-Tetrametilentetrahexahidropirimidina (TMTP) con Aminas Aromáticas $p$-sustituidas. Tesis de Grado, Química, Universidad Nacional de Colombia, 2001. 
[8] T.Cummings, J. Shelton, Chemistry Organic, 1960, 25, 419.

[9] M.Issa, A. Khedr, H. Rizk, Spectrochim. Act Part A., 2005, 62, 621.

[10] G. Santafe, Contribución al estudio de la reactividad del 1,3,6,8- tetraazatricilo[4,4,1,13,8]dodecano (TATD). Tesis de Maestría en Ciencias Químicas, Universidad Nacional de Colombia, 1994.

[11] F. F. Dos Santos, C. F. Tormena, Estructura Molecular, (THEOCHEM), 2006, 763, 145.

[12] M. Ladika, N. G. Rondan,. Molecular Structure. (THEOCHEM), 1996, 365, 21.
[13] J.M. Locke, R.I. Crumbie, R. Griffith, T.D. Bailey, S. Boyd, J.D. Roberts, organic Chemistry, 2007, 72, 4156.

[14] M. R. Silverstein, Spectrometric Identification of Organic Compound, $3^{\text {th }}$ Ed John Wiley \& Sons, Inc., 1974, p. 177- 181.

[15] G. I. Gallo, M. E. Gayon, Nuevos aportes al mecanismo de reacción de obtención de Bis-1,3-bonzoxacinas, Tesis de Grado,Farmacia, Universidad Nacional de Colombia, 1991.

[16] J. McMurrry, Química Orgánica" Ed. Thompson-Internacional Editores S.A. 2002, p. 365-367. 